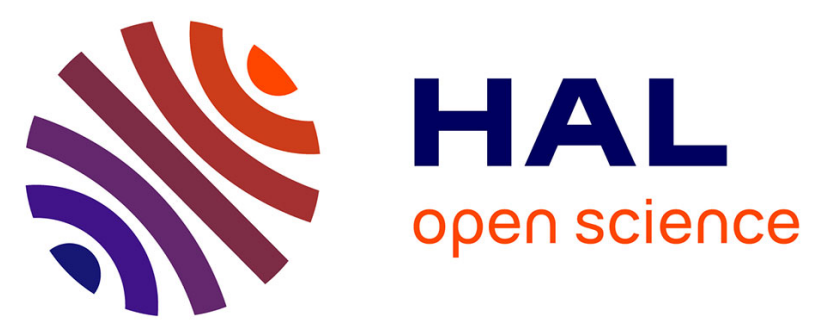

\title{
Interictal spikes, fast ripples and seizures in partial epilepsies - combining multi-level computational models with experimental data.
}

Fabrice Wendling, Fabrice Bartolomei, Faten Mina, Clémént Huneau, Pascal Benquet

\section{To cite this version:}

Fabrice Wendling, Fabrice Bartolomei, Faten Mina, Clémént Huneau, Pascal Benquet. Interictal spikes, fast ripples and seizures in partial epilepsies - combining multi-level computational models with experimental data.: computational and experimental models of epilepsy. European Journal of Neuroscience, 2012, 36 (2), pp.2164-77. 10.1111/j.1460-9568.2012.08039.x . inserm-00728701

\section{HAL Id: inserm-00728701 https://www.hal.inserm.fr/inserm-00728701}

Submitted on 6 Nov 2012

HAL is a multi-disciplinary open access archive for the deposit and dissemination of scientific research documents, whether they are published or not. The documents may come from teaching and research institutions in France or abroad, or from public or private research centers.
L'archive ouverte pluridisciplinaire HAL, est destinée au dépôt et à la diffusion de documents scientifiques de niveau recherche, publiés ou non, émanant des établissements d'enseignement et de recherche français ou étrangers, des laboratoires publics ou privés. 
Interictal spikes, fast ripples and seizures in partial epilepsies: combining multi-level computational models with experimental data

Running title: computational and experimental models of epilepsy

Fabrice Wendling $^{1,2}$, Fabrice Bartolomei $^{3,4}$, Faten Mina ${ }^{1,2}$, Clémént Huneau ${ }^{1,2}$, Pascal Benquet $^{1,2}$

${ }^{1}$ INSERM, U642, Rennes, F-35000, France

${ }^{2}$ Université de Rennes 1, LTSI, F-35000, France

${ }^{3}$ INSERM, U751, Marseille, F-13000, France

${ }^{4}$ AP-HM, Hôpital de la Timone, Service de Neurophysiologie Clinique, Marseille, F-13000, France

Corresponding author: fabrice.wendling@univ-rennes1.fr

Submitted to the European Journal of Neuroscience, section Neurosystems

Special issue on

"The Dynamics of Neurological Disease:

Integrating Computational, Experimental and Clinical Neuroscience"

Revised version (R1), January 9, 2012 


\begin{abstract}
Epileptic seizures, epileptic spikes and high frequency oscillations (HFOs) are recognized as three electrophysiological markers of epileptogenic neuronal systems. It can be reasonably hypothesized that distinct (hyper)excitability mechanisms underlie these electrophysiological signatures . The question is "Which are these mechanisms?". Solving this difficult question would considerably help our understanding of epileptogenic processes and would also advance our interpretation of electrophysiological signals. In this paper, we show how computational models of brain epileptic activity can be used to address this issue. With a special emphasis on the hippocampal activity recorded in various experimental models (in vivo and in vitro) as well as in epileptic patients, we confront results and insights we can get from computational models lying at two different levels of description, namely macroscopic (neural mass) and microscopic (detailed network of neurons). At each level, we show how spikes, seizures and HFOs can (or cannot) be generated depending on the model features. The replication of observed signals, the prediction of possible mechanisms as well as their experimental validation are described and discussed; as are the advantages and limitations of the two modeling approaches.
\end{abstract}

Keywords: epilepsy; models; computational; in vivo; in vitro; interictal spikes; fast ripples; seizures 


\section{Introduction}

Drug-resistant partial epilepsies refer to a group of chronic neurological disorders which cannot be treated pharmacologically. By definition, partial epilepsies are characterized by recurrent seizures generated in a focal or multi-focal zone relatively well circumscribed in the brain and often referred to as the epileptogenic zone (EZ). From the clinical point of view, the accurate delineation of the EZ is the essential diagnostic step in the perspective of surgery which is, to date, the only therapeutic option that can lead to a total suppression of seizures.

In some specialized Epilepsy Surgery Units, a direct exploration of brain regions is performed using intra-cerebral (i.e. depth) electrodes (stereo-EEG or depth-EEG recordings)(Bancaud \& Talairach, 1973). This method has long been considered as the "gold standard" for EZ identification. It provides data about both interictal and ictal brain activity with an excellent temporal resolution in the form of time series signals corresponding to local field potentials (LFPs) generated by explored regions.

Although the direct recording of LFPs from brain regions has dramatically advanced the assessment of the EZ, the demand is still high for diagnostic methods allowing for better interpretation of these signals, in terms of underlying pathophysiological mechanisms (Stefan et al., 2006).

Generally speaking, three main types of electrophysiological events are observed in depthEEG signals recorded from the epileptic brain, namely seizures, spikes and high-frequency oscillations (HFOs). In partial epilepsies, seizures involving temporo-mesial and/or neocortical structures are most often characterized by a tonic onset phase (so-called rapid discharges) followed by a clonic phase marked by a highly rhythmic activity synchronized across vast and distant regions. Rapid discharges (classically in the upper beta and gamma bands of the EEG, i.e. 25 to $100 \mathrm{~Hz}$ ) have long been considered as the hallmark of the EZ (Talairach \& Bancaud, 1966; Fisher et al., 1992; Wendling et al., 2003; Bartolomei et al., 
2008). Epileptic spikes have also been recognized as potentially useful markers of epileptogenic areas as these transient paroxysmal events are being generated in very early stages in experimental models of epileptogenesis (White et al., 2010) and are very often (if not always) observed during interictal periods in patients (review in (Rodin et al., 2009)). However, the diagnostic value of epileptic spikes remains controversial since their relation to seizures is not elucidated yet (Avoli et al., 2006; Staley et al., 2011) (Hufnagel et al., 2000; Asano et al., 2003; Marsh et al., 2010). Finally, over the past decade, the significance of HFOs in epilepsy, especially fast ripples (FRs), has been a topic of increasing interest (recent review in (Zijlmans et al., 2011)). FRs are transient low-amplitude fast oscillations (250-600 $\mathrm{Hz}$ ) observed in signals recorded with intracerebral electrodes. Seminal studies reported the presence of FRs in experimental models of epilepsy (Bragin et al., 1999b) as well as in the human epileptic brain (Bragin et al., 1999a). FRs were also hypothesized as unique to brain areas capable of generating spontaneous seizures (Jacobs et al., 2009) and their clinical value has been confirmed in recent papers (Jacobs et al., 2010; Worrell \& Gotman, 2011).

In the light of results reported in aforementioned studies, one can reasonably assume that seizures, epileptic spikes and HFOs correspond to distinct mechanisms of (hyper)excitability within underlying neuronal networks which generate observed electrophysiological signals. The question is "Which ones?". Solving this difficult question would considerably help our understanding of epileptogenic processes and would also advance our interpretation of the above-mentioned electrophysiological signatures of these processes.

In this paper, we address this issue through the use of computational models of brain epileptic activity developed in our team. But our intent is to go beyond the simple review of models we introduced over the past ten years. For the first time, we confront results and insights we got from such models lying at different levels of description, namely macro- and micro-scopic. At each level, we show how spikes, seizures and HFOs can (or cannot) be generated depending 
on the model features. With a special emphasis on the hippocampal activity, the replication of observed signals, the prediction of possible mechanisms and their experimental validation are described and discussed; as are the advantages and limitations of the two modeling approaches.

\section{Material and methods}

\section{Experimental data recorded from hippocampus}

The objective of this section is to present the electrophysiological features of epileptic events under study (epileptic spikes, FRs and seizures). The events shown in this study were extracted from electrophysiological data collected in animal models (in vivo, in vitro) and in a patient with temporal lobe epilepsy (TLE), as described below.

\section{In vivo data}

Experiments were conducted on $80 \pm 5$ days old C57BL/6J (B6) male mice. All animal procedures were conducted in accordance with the European Communities Council Directive of 24 November 1986 (86/609/EEC). Mice were stereotaxically injected under general anesthesia (chloral hydrate $400 \mathrm{ml} / \mathrm{kg}$ i.p.) with low dose of kainic acid (KA, $50 \mathrm{nl}$ of a 20 $\mathrm{mM}$ solution in $0.9 \% \mathrm{NaCl}$ ) into the right dorsal hippocampus (anteroposterior $=-2$, mediolateral $=-1.5$, dorsoventral $=-2 \mathrm{~mm}$ ) with bregma as reference according to Franklin $\&$ Paxinos atlas. Control mice were injected with saline solution under the same protocol. After intrahippocampal injection, all mice were implanted with a bipolar electrode inserted into the injected hippocampus (anteroposterior $=-2$, mediolateral $=-1.5$, dorsoventral $=-1.9 \mathrm{~mm}$ ) and two monopolar surface electrodes were placed over the right frontoparietal cortex and over the cerebellum (reference electrode). LFPs were recorded on a video-EEG monitoring system (Deltamed TM). Signals were sampled at $2048 \mathrm{~Hz}$ (16 bits). Upon completion of the 
experiments, histological analyses were performed to verify the location of the KA injection, the location of the hippocampal electrode and the pattern of neuronal loss/dispersion of dentate gyrus granule cells. This mouse model of TLE was previously described (Suzuki et al., 1995) to reproduce histological characteristics similar to those found in hippocampal sclerosis described in human mesial TLE, such as cell loss, granule cell dispersion, astrogliosis and mossy fiber sprouting (Bouilleret et al., 1999). Moreover, studies of epileptogenesis in this model revealed that sporadic epileptic spikes change as a function of time as the brain evolves towards the chronic epileptic state characterized by the repetition of spontaneous seizures (Huneau et al., 2010).

\section{Hippocampal organotypic slices}

Hippocampal slice cultures were prepared from 6-d-old Wistar rats as described previously (Gahwiler et al., 1997) following a protocol approved by the Veterinary Department of the Canton of Zurich. Briefly, hippocampi were dissected and transverse slices (375 $\mu \mathrm{m}$ thick) were maintained on a glass cover slip in individual tubes filled with culture medium. Each tube was then placed in a roller drum in an incubator at $36^{\circ} \mathrm{C}$ for 2 weeks. After 2-3 weeks in vitro slices were transferred to a recording chamber on an upright microscope (Axioscope FS, Zeiss, Oberkochen, Germany). Hippocampal slice cultures are a particularly valuable in vitro model to study some cellular mechanisms of epilepsy. Because of the inevitable damaging of axons during the preparation of acute brain slices (ex-vivo), many projection pathways are kept more intact in cultured slices as compared with ex vivo slices. Indeed, during the preparation of brain slice culture, the density of synapses first decreases just after the brain cut, but then synaptic structures gradually redevelop (in situ synaptogenesis). Therefore, the degree of connectivity between neurons in culture increases with time. It is more prominent than that of acute brain slices and it was shown to be closer to the in vivo situation (Gahwiler et al., 
1997). The hippocampal circuitry within these cultures is well preserved and is able to support synchronized network activity. The cellular network develops in such a way that synaptic inhibition and excitation are balanced appropriately (Thompson et al., 2005). Thus, the high degree of interneuronal and pyramidal connectivity in organotypic slice cultures, and the spontaneous synaptic activity, facilitate the induction of epileptic activity. Indeed, applications of convulsivants, including bicuculline, picrotoxin, tetraethylammonium (TEA), or low Mg2+or high $\mathrm{K}+$-containing saline, can trigger both interictal and ictal-like burst discharges that are synchronized throughout large cell populations (Scanziani et al., 1994). These bursts appear essentially identical to those evoked in vivo or in acutely prepared hippocampal slices (Thompson et al., 2005).

Both intracellular and extracellular recordings were performed to measure interictal- and ictallike activity in organotypic slices. Intracellular activity of CA1 pyramidal neurons was recorded in the whole cell configuration of the patch-clamp technique. Recording pipettes (3-6 $\mathrm{M} \Omega$ ) were filled with an intracellular solution containing the following: $135 \mathrm{mM} \mathrm{K}$-Gluconate, 4 mMKCl, 10 mM HEPES, 10 mM Na2-phosphocreatine, 4 mM Mg-ATP, 0.3 mM Na-GTP, pH 7.2, 291-293 mOsm. Simultaneous field recordings were obtained with an extracellular electrode (glass pipette filled with normal ACSF, $1 \mathrm{M} \Omega$ ) positioned in CA1 stratum radiatum. The field recording pipette was placed into the same area as the patch clamp pipette (4-6 M $\Omega$ ). Current-clamp mode was used and signals were amplified with an Axopatch 200B amplifier (Axon Instruments, Foster City, CA, USA), filtered at $2 \mathrm{kHz}$, stored and visualized with pClamp9 software (Axon Instruments). Signals were analog-filtered at $10 \mathrm{kHz}$ and sampled at 100-300 kHz. Stimulation (100 $\mu \mathrm{s}, 10-40 \mu \mathrm{A}$, every $10 \mathrm{sec})$ was applied with a glass pipette filled with artificial cerebrospinal fluid placed in CA3 pyramidal layer. When requested, recordings were performed continuously in gap-free mode in order to obtain an entire period of spontaneous ictal- and intercictal-like activity. Slices were superfused continuously at a rate of 
1-2 $\mathrm{ml} / \mathrm{min}$ with normal artificial cerebrospinal fluid (ACSF) equilibrated with $95 \% \mathrm{O} 2 / 5 \%$ CO2 containing (in mM): $124 \mathrm{NaCl}, 2.5 \mathrm{KCl}, 26 \mathrm{NaHCO} 3,1.25 \mathrm{NaH} 2 \mathrm{PO} 4,10$ glucose, 3 $\mathrm{CaCl} 2,2 \mathrm{MgCl} 2, \mathrm{pH} 7.4$, at a bath temperature of $34^{\circ} \mathrm{C}$.

\section{Guinea pig isolated brain preparation}

For the purpose of this study, we used data recorded from an isolated brain preparation which is an acute model of temporal lobe ictogenesis (courtesy of Dr M. de Curtis, Istituto Neurologico Carlo Besta, Milan, Italy). Brains of Hartley guinea pigs (150-200g, Charles River, Calco, Italy) were isolated and maintained in vitro according to the standard procedure described elsewhere (Muhlethaler et al., 1993; Avoli et al., 2006). In brief, animals were anesthetized with sodium tiopental $(125 \mathrm{mg} / \mathrm{Kg}$ i.p., Farmotal, Pharmacia) and transcardially perfused with a cold $\left(4-10^{\circ} \mathrm{C}\right)$ oxigenated $\left(95 \% \mathrm{O}_{2}, 5 \% \mathrm{CO}_{2}\right)$ complex saline solution composed of $126 \mathrm{mM} \mathrm{NaCl}, 3 \mathrm{mM} \mathrm{KCl}, 1.2 \mathrm{mM} \mathrm{KH}_{2} \mathrm{PO}_{4}, 1.3 \mathrm{mM} \mathrm{MgSO}_{4}, 2.4 \mathrm{mM} \mathrm{CaCl}_{2}$, $26 \mathrm{mM} \mathrm{NaHCO} 3,15 \mathrm{mM}$ glucose, $2.1 \mathrm{mM}$ HEPES and 3\% dextran M. W. 70000 (pH=7.1). Brains were rapidly dissected out and transferred in the recording chamber where they were perfused through the basilar artery with the same solution $\left(5.5 \mathrm{ml} / \mathrm{min}, \mathrm{pH}=7.3,15^{\circ} \mathrm{C}\right)$. The experiments were performed at $32^{\circ} \mathrm{C}$ after gradually raising the temperature by steps of $0.2^{\circ} \mathrm{C} / \mathrm{min}$. Extracellular recordings were performed in the hippocampus (CA1 region) with 16-channels silicon probes (100 $\mu \mathrm{m}$ contact separation, CNCT, University of Michigan, Ann Arbour, MI). Probes were inserted in the tissue as perpendicular as possible to the lamination of the structure according to a guinea-pig stereotaxic atlas. At the end of the electrophysiological experiments, electrolytic lesion were made by passing a $30 \mu \mathrm{A}$ current for 30 seconds between the two deepest contacts of the silicon probes. Brains were then fixed and cut with vibratome in $75-100 \mu \mathrm{m}$ thick coronal sections to verify the position of the 
electrodes. The experimental protocol was reviewed and approved by the Committee on Animal Care and Use and by Ethics Committee of the Istituto Nazionale Neurologico.

\section{Clinical data}

The intracerebral stereo-EEG (SEEG) recording used in this study was performed in a patient with mesial TLE. For simplicity, only signals recorded from contacts located in the hippocampus (Amon's horn) are shown and analyzed in the present study. It is worth mention that these signals are very representative of those recorded from the hippocampus in mesial TLE. Signals were recorded on a 128-channel Deltamed ${ }^{\mathrm{TM}}$ system and were sampled at 1024 Hz. The positioning of electrodes was determined from available non-invasive information and hypotheses about the localization of the patient's epileptogenic zone. Implantation accuracy was per-operatively controlled by telemetric X-ray imaging. A post-operative CT scan without contrast product was used to verify both the absence of bleeding and the precise 3D location of electrode contacts. After SEEG exploration, intracerebral electrodes were removed, and an MRI was performed on which the trajectory of each electrode remained visible. Finally, a CT-scan/MRI data fusion was performed to anatomically locate each contact along each electrode trajectory. The SEEG exploration was carried out as part of normal clinical care of the patient who gave informed consent about the use of data for research purposes.

\section{Electrophysiological features of epileptic spikes, FRs and seizures}

Sporadic epileptic spikes could be observed in above-described experimental models (in vivo and in vitro), as well as in depth-EEG signals recorded from patient with mesial TLE. Typical examples are shown in Fig.1.A. As depicted (first three boxes), these spikes recorded from hippocampus have common morphological features. Their onset is characterized by an initial fast component (the spike) followed by a slow negative (w.r.t. baseline) wave. The duration 
(from onset to baseline return) is of the same order of magnitude (about one or two hundreds of $\mathrm{ms}$ ) in the three considered situations (mouse in vivo, rat in vitro and human). As described in the Results section, we could reproduce the spike shape, both in the lumped-parameter and in the detailed model of hippocampus (Fig.1.A, fourth and fifth box), which are both presented in the next section.

FRs were also observed in real data (experimental and clinical) as shown in Fig.1.B. As revealed by time-frequency representations (spectrograms in Fig.1.B), FRs are transient events characterized by low-amplitude high-frequency oscillations occurring in a frequency sub-band ranging from $250 \mathrm{~Hz}$ to $600 \mathrm{~Hz}$ (approximately). Some similarities could also be found across experimentally or clinically recorded FRs, but to a lesser extent than epileptic spikes which really have a stereotyped shape. In particular, their duration is about a few tens of ms. As also described in the results, we recently managed to simulate FRs in a detailed model of hippocampal activity (presented in the next section). Interestingly, such a simulation could not be obtained when the lumped-parameter modeling approach was followed.

Finally, electrophysiological signals recorded during the transition from interictal to ictal activity in experimental models of epilepsy and in the considered patient are displayed in Fig.2. As depicted, the general time-course of electrophysiological signals recorded in human (Fig. 2.A), in the epileptic freely-moving mouse (Fig.2.B) and in the isolated Guinea-pig brain (Fig. 2C) is quite reproducible. Indeed, these signals are very typical of those recorded in mesial TLE, particularly from the hippocampus. Usually, interictal and preictal spikes are observed before the onset of the seizure marked by the appearance of higher frequency oscillations $(20-30 \mathrm{~Hz})$ which maintain for a few seconds (typically 5-10 s). This early tonic phase of the seizure is then followed by a clonic phase characterized by the appearance of a slower and very rhythmic activity $(5-10 \mathrm{~Hz})$ of larger amplitude. So far, as illustrated in Fig. 2.D, we could reproduce the above-described features in the lumped-parameter model 
(described in the next section). Finally, Fig. 2.E provides time-frequency representations focused on the onset of seizures for clinical, experimental and simulated data. As observed, the fast onset activity is always present (white arrows in spectrograms). Its duration is reduced in the mouse (about $1 \mathrm{~s}$ ).

\section{Computational models}

Computational neuroscience is an interdisciplinary field of research at the interface of neurophysiology, neurobiology, mathematics and physics. In the field of epilepsy, computational modeling approaches are considerably developed and models are more and more accepted by clinicians and research scientists as they provide an efficient way to gather information into manageable representations of neuronal systems, to articulate knowledge and to bridge between disciplines (Lytton, 2008; Soltesz \& Staley, 2008; Wendling, 2008). Models are also recognized as efficient tools for tackling the complexity of epileptic phenomena since they can account for the many nonlinear mechanisms taking place at multiple levels in neuronal systems, from sub-cellular to organ levels. Over the past three decades, two computational modeling approaches have been developing, either at the lumped (neural mass) or detailed (network of neurons) level. Readers may refer to (Suffczynski et al., 2006; Deco et al., 2008; Ullah \& Schiff, 2009; Coombes, 2010) for background and details about the concepts which led to both modeling approaches. In brief, the central issue is the scale at which the neuronal population is considered. According to the lumped-parameter approach (macroscopic level) which stems from pioneering work of Wilson and Cowan (Wilson \& Cowan, 1972), Freeman (Freeman, 1973) and Lopes da Silva (Lopes da Silva et al., 1974), the neuronal population is assumed to be composed of sub-populations of cells (typically main cells and interneurons) interacting through synaptic transmission. This approach aims at describing the "average activity" of the population without explicit representation of signals generated at the cellular level (typically action potentials - APs - and 
single post-synaptic potentials - PSPs). This is why the two relevant variables in lumpedparameter models are i) the average density of APs (i.e. firing rate) and ii) the average PSPs (either excitatory or inhibitory) generated at the level of each sub-population included in the whole population. Conversely, the detailed approach starts from a finer description of neurons which are then interconnected via synapses or gap junctions to form networks. This description accounts for the active and passive properties of the excitable membrane of neurons. It can be either simple (one compartment) or more sophisticated (i.e. multicompartmental) if dendrites, soma and axons are to be accurately represented for instance. Most of the detailed models are still based on the Hudgkin and Huxley's mathematical equations governing transmembrane currents via voltage-dependant ion channels (Hodgkin \& Huxley, 1952; Hodgkin et al., 1952). In the field of epilepsy, the detailed approach started with Traub's work (Traub, 1979) and progressively developed, partly helped by the tremendous increase of computer performances (computing time and memory) since the eighties.

\section{A lumped-parameter model of the hippocampal CA1 circuit}

Following the lumped-parameter approach, we developed a model for the hippocampal CA1 subfield. Readers may refer to (Wendling et al., 2002; Wendling et al., 2005) for details. Briefly, the neuronal population of CA1 is assumed to consist of three sub-populations of neurons corresponding to main (pyramidal) cells and to two types of local inhibitory interneurons (oriens-lacunosum moleculare - OLM - and basket cells) projecting to either the peri-dendritic or the peri-somatic region of pyramidal cells, as described in (White et al., 2000; Cossart et al., 2001). An inhibitory connection from OLM to basket cells is also represented in the model (White et al., 2000) as depicted in the schematic diagram provided in Fig. 3.A. Input to interneurons is excitatory (glutamate receptor-mediated). Feedback to 
pyramidal cells is either excitatory (glutamate receptor-mediated) or inhibitory (GABA receptor-mediated). Slow $\left(\mathrm{GABA}_{\mathrm{A}, \text { slow }}\right)$ and fast $\left(\mathrm{GABA}_{\mathrm{A}, \text { fast }}\right)$ kinetics are associated to inhibitory post-synaptic potentials (PSP) depending on the location of GABA receptors, either in the peri-dendritic or in the peri-somatic region of pyramidal cells (Banks et al., 2000; Klausberger \& Somogyi, 2008). In each sub-population, the mean membrane potential is converted into an average pulse density of potentials fired by the neurons using a static nonlinear function (asymmetric sigmoid curve), referred to as the "wave-to-pulse" function. Conversely, the average pulse density of afferent action potentials is changed into an average inhibitory or excitatory post-synaptic membrane potential using a linear dynamic transfer function. The impulse response of this "pulse-to-wave" function was shown to approximate that of actual postsynaptic potentials (van Rotterdam et al., 1982). In the model, these "pulseto-wave" functions introduce the three main parameters which respectively correspond to the amplitude of average i) excitatory PSPs $(E X C)$, ii) slow dendritic inhibitory PSPs $(S D I)$ and iii) fast somatic inhibitory PSPs $(F S I)$. Besides, the model also accounts for the average number of synaptic connections among the three sub-populations. Finally, the model output corresponds to the summation of these average PSPs on pyramidal cells which is assumed to be the principal contribution to local field potentials. A block diagram of the model as well as the value and the meaning of each model parameter are provided in (Wendling \& Chauvel, 2008b)(see figure 23.12 and table 23.3).

\section{A detailed model of the hippocampal CA1 network}

More recently and following the detailed approach, we developed a neuronal network model for the hippocampal CA1 subfield (Demont-Guignard et al., 2009). We started by developing a two-compartment 'reduced' model of the CA1 pyramidal cell in line with Pinsky and Rinzel's work on CA3 cells (Pinsky \& Rinzel, 1994). This single neuron model is minimal 
but still biologically-inspired. The objective was to find a good compromise between physiological relevance and computing time. Based on the Hodgkin and Huxley formalism (Hodgkin \& Huxley, 1952; Hodgkin et al., 1952), the model entails distinct somatic and dendritic membrane properties. Transmembrane currents were selected based on a review of the literature. A validation was performed using intracellular data recorded in an experimental model (organotypic rat hippocampal slices). A schematic diagram of the neuron model is shown in Fig. 3.B. In brief, the shape of the action potential (AP) is mainly controlled by voltage-dependent sodium, potassium and calcium currents $\left(I_{N a}, I_{K D R}, I_{C a}\right.$, low and high threshold). In addition, to replicate physiological firing rates potassium channels activated by intracellular calcium ions were added (after-hyperpolarisation current $I_{A H P}$ ). Similarly, we also included a rapidly inactivated potassium current $\left(I_{K A}\right.$, dendritic compartment (Hoffman et al., 1997) to reproduce the change of density of ion channels along the membrane) in consideration of its potential role in epileptiform activity, as well as a hyperpolarizationactivated cationic current ( $I_{h}$, dendrite compartment (Mayer \& Westbrook, 1983)) and a muscarinic potassium current ( $I_{m}$, (Lancaster \& Adams, 1986; Lancaster \& Nicoll, 1987)). Regarding basket and OLM interneurons, we implemented single-compartment models published in the literature (Hajos et al., 2004). Single neuron and interneuron models were interconnected via glutamatergic (AMPA and NMDA) and GABAergic synapses in accordance with commonly accepted assumptions about location of synapses and receptors (somatic vs. dendritic) and connectivity patterns in CA1 (Andersen et al., 2007). Finally, the network model is stimulated with an afferent volley of APs coming from an "external array of cells" (Fig. 3.B). The objective was to mimic the excitatory input from CA3 to CA1. In practice, this stimulation of the CA1 network depends on two parameters: i) the number of cells, randomly and uniformly selected in CA1, that are contacted by axons firing action potentials and ii) the value of the delay between these action potentials which follows a 
Gaussian distribution which mean and standard deviation allow for adjusting the synchronicity of APs.

Regarding the model output, we used the dipole theory for computing the LFP generated by the network. According to this theory, field signals due to individual neurons and recorded at a distance from the electrode are proportional to the magnitude of the net intracellular current dipole in each contributing cell (Geselowitz, 1967; Murakami \& Okada, 2006). To proceed, we made three assumptions. First, the main contribution to the LFP comes from pyramidal cells due to their spatial arrangement 'in palisades'. Second, each pyramidal cell is considered as a point source (current dipole formed by a sink and a source) positioned within a volume conductor characterized by a homogeneous conductivity. Third, according to the superposition theorem, the contributions of all pyramidal cells sum up instantaneously at the level of the extracellular recording electrode, placed at the center of the modeled network. For each cell, this contribution depends on the cell-electrode distance as well as the associated dipole moment and orientation.

Lumped-parameter and the detailed model of the hippocampal CA1 network: implementation details

For the macroscopic model, equations are provided in previously published papers (Wendling et al., 2002; Wendling et al., 2005). A didactic presentation of this class of models can also be found in a book chapter (Wendling \& Chauvel, 2008a). In addition, the source code can be downloaded from the Yale Model Database (http://senselab.med.yale.edu/modeldb/showmodel.asp?model=97983). For the microscopic model, equations are fully described in the two-pages appendix published in (DemontGuignard et al., 2009). 


\section{Results}

A first requirement in the development of models is to reproduce the observed phenomena, typically an extracellular activity which closely resembles that actually recorded. In the case of epileptic spikes, the "close resemblance" means that the simulated events should have the same morphological features (sharpness and the spike, duration of the negative wave, in particular) as real spikes. For FRs, simulated signals should contain oscillations occurring in the "correct" (i.e. actually observed) frequency band $(250-600 \mathrm{~Hz})$. For seizures, models should reproduce the fast onset activity observed during the initial tonic phase as well as the slower activity recorded during the clonic phase. A second requirement is that model parameters for which a more or less accurate replication of actual signals is obtained should stay in "physiological" ranges of values. But, this capability to replicate observations is not sufficient to guarantee that the mechanisms embedded in the model are those actually occurring in real neuronal systems. In Marder's group (Taylor et al., 2009), it has been recently shown that around 1300 different configurations of a four-compartment computational model (out of $\sim 600,000$ ) were able to well approximate the electrophysiological properties of a biological neuron, as simple as the LP neuron of the stomatogastric ganglion of decapod crustaceans. Thus mimicking and replicating the interictal or ictal-like activity of an epileptic neuronal network by a computational modeling is not sufficient. In all cases, further model validation is also required. This validation can be obtained by translating model predictions into experimentally testable hypotheses and, of course, by performing the tests. At least some of the predictions of the computational model have to be experimentally tested and validated.

In the following sections, we report the results we could get from the above-described models in terms of generating spikes, FRs or seizures. Then, we show examples for which some 
experimental validation of model-based assumptions on underlying mechanisms could be obtained.

\section{Model predictions}

Lumped-parameter approach. As mentioned in the methods section, the macroscopic model is characterized by three main parameters related to synaptic transmission: i) average excitatory PSPs $(E X C)$, ii) average slow dendritic inhibitory PSPs $(S D I)$ and iii) average fast somatic inhibitory PSPs $(F S I)$. We conducted a parameter sensitivity analysis in the 3D-space defined by (EXC, SDI, FSI), as reported in (Wendling et al., 2002). The basic procedure is i) to perform exhaustive simulations when the three parameters vary over physiological ranges of values step-by-step and ii) to classify segments of simulated activity obtained for each triplet based on spectral features. Six different classes were found: background activity, sporadic spikes, rhtythmic spikes, fast onset activity, ictal-like activity (alpha range) and ictallike activity (theta range). This procedure allowed us to map the activity generated by the model into the 3D-space corresponding to excitation- and inhibition-related parameters.

Results showed that the model (Fig 3.A) was able to generate transient events that closely resemble those recorded either in the human hippocampus or in animal models (in vivo and in vitro), as shown in Fig. 1.A, fifth column and in Fig. 4.B. The simulated epileptic spike is composed of a first and relatively sharp peak of positive polarity (w.r.t. baseline) followed by a slower wave of negative polarity. It was obtained under two conditions: parameter $E X C$ must be increased and parameter SDI must be decreased, indicating that epileptic spikes occur in the model for i) increased collateral excitation among pyramidal neurons and increased excitatory drive onto interneurons and ii) decreased inhibitory drive onto pyramidal neurons from dendritic-projecting interneurons (slow $\mathrm{GABA}_{\mathrm{A}}$ receptor-mediated IPSPs). The model was also found to generate a fast activity (around $25 \mathrm{~Hz}$, see Fig.4.C) mimicking that observed at the onset of seizure in the hippocampus, either in patients or in animal models of 
TLE (Fig. 2). This fast onset activity could be obtained when parameter SDI was decreased again, to a large extent. As illustrated in Fig.4.C, this important drop of parameter SDI led to a situation where i) somatic-projecting projecting interneurons (producing fast $\mathrm{GABA}_{\mathrm{A}}$ receptor-mediated IPSPs) were not inhibited anymore and ii) they still received excitatory drive from pyramidal cells. Finally, a clonic ictal-like activity could also be reproduced when a slight re-increase of parameter SDI occurred in the model whilst parameter FSI decreased. Two types of ictal-like activity could be simulated with a dominant frequency either around $10 \mathrm{~Hz}$ or around $5 \mathrm{~Hz}$ (Fig.4.D). A strong resemblance with the actual clonic activity recorded in the hippocampus was noticed (see Fig.4.D vs. Fig.2). Interestingly, we were not able to produce very HFOs in this lumped-parameter model, typically beyond the gamma frequency $(>80 \mathrm{~Hz})$. Some fast oscillations could be produced by modifying the rise and decay times of average PSPs but to unrealistic values, i.e. much lower than those characterizing actual glutamatergic or GABAergic PSPs. This result suggests that the simulation of FRs is impossible to achieve in our model under the constraint that physiological values for timerelated parameters (rise and decay time values of PSPs) are respected.

Detailed approach. As mentioned in the methods section, we built our own CA1 pyramidal cell (pc) model, after a accurate selection of dendritic and somatic ionic currents (and associated conductances) from the literature. This single pyramidal neuron model was evaluated by comparing the simulated intracellular activity with real intracellular activity recorded from pyramidal cells in the in vitro model. Taking advantage of the abundant literature about the neurophysiological properties of CA1 pyramidal cells, we tried to respect as much as possible some of the physiological properties of ionic channels such as the kinetics of activation and inactivation, the voltage dependence and the reversal potentials. This single cell model, although reduced to two compartments, and extremely simplified compared to a real neuron, was found to generate fairly realistic signals, as reported in 
(Demont-Guignard et al., 2009). Qualitatively, our CA1 pc model is able to reproduce basic electrophysiological properties of real CA1 pyramidal cell such as action potential (AP) shape and threshold, spike rate adaptation upon depolarizing current, sag plus rebound upon hyperpolarization and increase in spiking rate upon $\mathrm{I}_{\mathrm{KA}}$ inhibition (Demont-Guignard et al., 2009). The CA1 network was built by connecting these CA1 pc cells in accordance with commonly accepted assumptions about connectivity patterns (location of synapses and receptors, synaptic strength, inter-cell distances). The percentage of pyramidal cells was set at $80 \%$, that of interneurons at $20 \%$, as in vivo (Andersen et al., 2007). Two main differences between detailed and lumped models have to be pointed out: 1) the detailed model allows for topological repartition of individual pyramidal cells and for possible asynchrony in the activity of simulated neurons, 2) the detailed model can simultaneously simulate both the extracellular LFP and the intracellular activity of virtually thousands of individual cells. Thanks to these two properties, we have found that FRs are generated by small clusters of hyperexcitable and slightly asynchronous bursting pyramidal cells surrounded by hyperpolarized neurons, as shown in Fig.5.B. The origin of the hyperexcitability was due to a moderate increase of glutamatergic conductances (AMPA and NMDA), to a slight decreased conductances associated with GABAergic currents, and to a shift of the GABA reversal potential toward more depolarized values (Demont-Guignard et al., 2011). The low number of hyperexcitable neurons, the degree of synchronicity and the network topology (clustered) was found to be critical to produce FRs around $250 \mathrm{~Hz}$ and up to $500 \mathrm{~Hz}$. The model was also able to reproduce epileptic spikes relatively similar to human or animal epileptic spikes (Fig. 1), again not spontaneously but for a physiologically relevant stimulation mimicking the input from CA3 to CA1, reminding the "CA3-driven interictal activity" (Avoli 2002). The model could generate epileptic spikes for a sufficiently large subset (i.e., $>30 \%$ of the total number of cells) of quasi-synchronously firing CA1 pyramidal cells. To produce realistic epileptic 
spikes the reversal potential for $\mathrm{GABA}_{\mathrm{A}}$ receptor has also been modified to more depolarized values in some pyramidal cells (Demont-Guignard et al., 2011).

Overall, the number and spatial distribution of involved pyramidal cells, their degree of excitability and level of synchronization are critical factors that strongly modify the morphology of the induced epileptic event that reflects in the LFP (Fig.5, A, B and C). Interestingly, as previously reported (Demont-Guignard et al., 2011), a continuum between "normal" population spike, FR and epileptic spike could be found depending on synaptic transmission parameters. For increase of the AMPA and NMDA conductances and decrease of the GABA conductance, simulated events progressively shifted from normal population spikes to FRs, from FRs to IESs, and then from IESs to ictal-like activity (not shown). As presented in the next section, this model prediction could be verified experimentally.

\section{Model validation}

Lumped-parameter approach. An interesting prediction of the lumped-parameter model is related to the generation of a fast activity which satisfactorily approximates that observed at the onset of seizures in TLE (human data), in an experimental model of TLE and in an experimental model of temporal lobe ictogenesis (guinea pig), as illustrated in Fig.2. In the computational model, this fast activity is obtained when i) dendritic-projecting interneurons do not generate slow IPSPs $\left(\mathrm{GABA}_{\mathrm{A}}\right.$ receptor-mediated) anymore and ii) somatic-projecting projecting interneurons still receive some excitatory drive from pyramidal cells and, in turn, generate fast IPSPs $\left(\mathrm{GABA}_{\mathrm{A}}\right.$ receptor-mediated) onto pyramidal cells. In other words, and as already reported in (Wendling et al., 2002), the model predicts i) a crucial role of inhibitory networks during the transition to seizures in the hippocampus and ii) a prominent contribution of the fast inhibitory feedback loop in the generation of high-beta, low gamma (20 to $30 \mathrm{~Hz}$ ) activity, as quantified in the simulated LFP. To some extent, an experimental validation of these modeling results was recently brought by M. de Curtis and colleagues (Gnatkovsky et 
al., 2008) although these authors focused on the enthorinal cortex (EC) which is also a limbic structure frequently involved in human TLE (Molaee-Ardekani et al., 2010a). The objective of these authors was to analyze the cellular and network mechanisms underlying the generation of $20-30 \mathrm{~Hz}$ oscillations recorded at the onset of TLE seizures. First, the in vitro isolated guinea pig brain, they could reproduce such a pattern in the EC which is quite similar to that observed in the hippocampus (see example in Fig. 2.C and 2.E), i.e. a narrow band activity characterized by a dominant frequency at about $25 \mathrm{~Hz}$. During the transition to seizure induced by a transient arterial perfusion of bicuculline, they performed simultaneous intracellular and extracellular electrophysiological recordings in the EC (deep and superficial layers). As a major result, they observed that principal neurons did not generate action potentials during the fast onset activity observed in the LFP whilst a sustained firing could be revealed in non-principal neurons which were likely to be interneurons (so-called "putative" interneurons). In addition, during the fast activity, they could detect some rhythmic oscillations in the membrane potential of principal neurons. Results strongly suggested that these oscillations were due to rhythmic IPSPs that progressively decreased in amplitude as the fast onset activity progressively changed into the slower clonic activity. Coming back to the lumped-parameter model, we analyzed the firing rates of the sub-populations of neurons during the simulated transition from interictal to ictal activity obtained for gradual variation of the SDI parameter, as described in the result section. Very interestingly, the model showed that the average firing rate at the pyramidal cell sub-population increased during pre-ictal spikes, dramatically decreased during the fast activity before increasing again as the ictal clonic activity started. It is also worth mention that a strong increase of the average firing rate of the sub-population of interneurons (producing fast IPSPs onto pyramidal cells) was generated by the model (Fig. 6), corroborating above-described experimental observations. 
Detailed approach. As mentioned in the methods section, we used rat organotypic hippocampal slices to perform experimental validation because they offer a number of advantages among which the preserved connectivity of both excitatory and inhibitory neurons. In these slices, depending on experimental procedure, we could produce normal field potentials, epileptic spikes, FRs, or ictal-like activity. For example application of 4-AP $(100 \mu \mathrm{M})$ produced both interictal and ictal-like activity in $98 \%$ of the slices $(n=34$, data not shown), as already observed in (Albus et al., 2008). Simultaneous intracellular (patch-clamp whole-cell recordings) and extracellular (field recordings) were performed in the CA1 region (Fig. 7.A). To reproduce the conditions used in the detailed computational model, we stimulated the CA3 area and recorded responses intracellularly from the CA1 pyramidal layer and extracellularly from CA1 stratum radiatum. When superfused with control ACSF, stimulation of CA3 axons induced a field postsynaptic potential (Fig.7B), through release of glutamatergic neurotransmitters (AMPA/NMDA). To follow the prediction of the detailed computational model, we complemented the above-described stimulation (activation of AMPA receptors and NMDA receptors) i) by a reduction GABA-current by using low concentration of GABA receptor antagonist Bicuculline and ii) by an indirect increase of NMDA current (by lowering the extracellular magnesium concentration from 1.2 to $0.3 \mathrm{mM}$ ). These procedures led to a fairly reproducible induction of FRs in LFP recordings (Fig.7C, top trace), associated with a strong slow depolarization (on top of which APs were produced) in simultaneous intracellular recordings (Fig. 7.C, bottom trace). During this stimulation protocol, the further increase of the slice excitability through the activation of AMPA receptors (supplementary bath application of a low dose of AMPA) led to the generation of epileptic spikes as predicted by the computational model (Fig. 7.D). Finally, we could also observe that the additional increase of the excitability of the slice (bath application of 4-AP 
$(50-100 \mu \mathrm{M})$ or high concentration of the $\mathrm{GABA}_{\mathrm{A}}$ receptors antagonist (Bicuculine $\left.50 \mu \mathrm{M}\right)$ led to the generation of ictal-like activity (Fig. 7.E).

\section{Discussion and perspectives}

The imbalance between excitation and inhibition has long been a leading concept in epilepsy research (Bernard et al., 1998). According to this concept, the epileptic tissue is characterized by either an increase of excitation or a decrease of inhibition at the level of principal cells, or both. This characteristic property of the epileptic tissue is probably true. However, we think that the excitation/inhibition concept is somehow too simplistic as a plethora of mechanisms most often nonlinear and highly-interlinked can lead to hyperexcitability in neuronal systems. We also think that this is precisely on this issue that computational models can help epilepsy research. Indeed, biologically-inspired models provide a very efficient framework to integrate many of these mechanisms into a system of constraints based on neurophysiological knowledge. In addition, when these computational models are intimately linked with experimental models, not only those mechanisms potentially leading to epileptic activity can be more easily identified but also a finer interpretation of observations can be derived. This model-based interpretation could progressively lead to a new era where our way of looking at electrophysiological signals (LFPs, EEG) would be changed and would get closer to underling mechanisms. More particularly, in the context of typical epilepsy-related signatures (interictal spikes and FRs, seizures) analyzed in this paper, we could get a number of insights regarding hyperexcitability from both macro- and micro-scopic computational models. These insights are discussed below, from a comparative perspective. Then, the limitations of the present study are addressed and some perspectives to this work are presented.

Hyperexcitability mechanisms in spikes, FRs and seizures: what do we learn from models? 
In the macroscopic (lumped) model, excitatory processes must definitively be increased and inhibitory processes must be decreased in order to produce interictal spikes and ictal activity. Increased excitation was achieved by increasing the amplitude of average EPSPs at each glutamatergic synaptic transmission site in the model (pyramidal to pyramidal cells, pyramidal cells to interneurons). Regarding inhibition, the situation is not as simple. The model predicted that $\mathrm{GABA}_{\mathrm{A}}$ receptor-mediated inhibition is a crucial factor as reviewed in (Avoli \& de Curtis, 2011) but also that two types (slow and fast) of inhibition (possibly corresponding to dendritic and somatic inhibition) were necessary to reproduce the typical phases and the corresponding signal dynamics observed during the interictal to ictal transition. Inhibition had to be indeed gradually lowered in the model to get to seizure

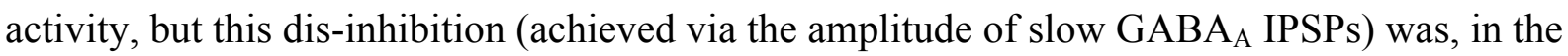
model, dendritic. The faster peri-somatic inhibition (fast GABA IPSPs) had to be maintained at seizure onset in order to produce fast oscillations in the simulated LFP. It is worth mentioning that some recent experimental findings support the hypothesis according to which the fast onset activity is closely related to the generation of fast IPSPs onto pyramidal cells (Gnatkovsky et al., 2008). An interesting finding in the macroscopic model is the impossibility to generate FRs under the constraint that rise and decay times of PSPs stay in appropriate physiological ranges. This model "incompleteness" can be explained by several properties of macroscopic models, among which the fact that individual cells (and thus firing patterns) are not explicitly represented. Indeed, this type of model assumes a hypersynchronisation of the activity of cells "merged" together into a given sub-population. This result indicates that this hypersynchronisation assumption is probably correct for the spiking, the fast onset $(20-30 \mathrm{~Hz})$ and the rhythmic ictal activity and that it may not hold when high frequency oscillations like FRs $(250-500 \mathrm{~Hz})$ are to be generated. Recently, we studied "chirp-like" rapid discharges $(70-100 \mathrm{~Hz})$ observed in neocortical seizures using a 
macroscopic model similar to that presented here but adapted to the cerebral cortex (MolaeeArdekani et al., 2010b). We noticed that it was somehow difficult to go beyond $110-120 \mathrm{~Hz}$ while respecting physiological values for the kinetics of EPSPs and IPSPs.

In the microscopic (detailed) model, we do not have, at this stage, many results regarding the simulation of the interitcal to ictal transition (see limitations below). This work-in-progress issue will be addressed in future studies. Regarding the generation of interictal spikes and FRs, we learnt that the excitation/inhibition ratio should also be increased to produce electrophysiological events resembling those actually observed. But here again, our results show that there are multiple manners to alter this ratio and that subtle changes in the hyperexcitability of simulated neuronal networks can lead to either spikes or FRs. In particular, the number of hyperexcitable cells (small vs. large clusters) is a first determinant factor. In our model, the fact GABAergic currents may become depolarizing is also an important factor in the generation of FRs and, to some extent, in the generation of spikes. This result corroborates those reported in (Cohen et al., 2002) and showing that depolarizing GABAergic events in subicular pyramidal cells contribute to interictal-like activity in an in vitro human subiculum preparation. The weak synchronization of firing patterns of pyramidal cells in the generation of FRs is also an interesting prediction of the model which corroborates recently published findings (Ibarz et al., 2010). Finally, our conductance-related parameter sensitivity analysis revealed that subtle changes in the synaptic transmission (increase of g_AMPA and g_NMDA, decrease of g_GABA) could lead to different electrophysiological events (population spikes, interictal-like spikes, FRs or sustained seizure-like activity) in the LFP. This prediction, which was experimentally verified, is interesting as the increase of g_AMPA can be interpreted in different ways. At post-synaptic level, it can correspond to changes in the properties of AMPA receptors (subunit alteration, phosphorylation, etc...) or to an increased number of channels (insertion of new channels, synaptic plasticity, LTP). At pre- 
synaptic level, it can describe an increased neurotransmitter release. All these mechanisms are encountered in the epileptic brain.

Macroscopic or microscopic?

Although epilepsy is a complex dynamical disease, it is generally admitted that epileptic activity corresponds to caricatured quasi-deterministic mechanisms. For this reason, we think that computational modeling is particularly adapted to epileptic processes because these processes are much less subtle than those involved in normal brain activity. To model epilepsy-related mechanisms, both macro- and micro-scopic approaches have their own advantages and limitations. The structure of macroscopic models as well as their parameters can be relatively easily adapted to the specific cyto-architecture of brain structures (for instance, the entorhinal cortex (Labyt et al., 2007)). They make it possible to simulate quite extended neuronal systems like the olfactory system (Freeman, 1978) or the thalamo-cortical loop (Suffczynski et al., 2001), among others. The recent past years have witnessed a considerable increase of interest for such approaches. This is probably because epilepsy is a disease which often involves relatively extended areas or systems that can hardly be represented at cellular level, given the still limited power of computers for simulating large scale neuronal networks with explicit representation of all neurons. However, parameters governing these models are themselves macroscopic and are not directly related to cellular or sub-cellular levels, by definition. In addition, they assume a synchronous activity of cells in the sub-populations interconnected to model the considered neuronal population. Our results show that this assumption may not always hold for all epileptic events. Therefore, we also need more detailed approaches that provide access to cellular parameters among which some are hardly accessible experimentally (like the proprieties of voltage-dependant or leak channels, ionotropic channels, ...). A nice feature of detailed models is also to simultaneously generate intra-cellular and extra-cellular activity, for all cell types represented in the network. 
However, difficulties in parameter identification increase with the level of detail, in particular regarding the network topology (Dyhrfjeld-Johnsen et al., 2007). In addition, there is an inherent compromise between building more and more detailed models versus being able to compute them in short time and using them in practical situations. To give an idea, in the detailed hippocampus model, each pyramidal cell is represented by a two-compartment model accounting for 16 different ionic currents (each based on Hodgkin-Huxley formalism). We simulated networks including up to 3000 cells and also accounting for three types of synapses (AMPA, NMDA and GABA). In such networks, the time required to generate 1 second of activity on a quad-core PC is about half an hour. In addition, the number of parameters is quite high and appropriate strategies must be defined to "globally" (i.e. at the level of all cells and synaptic interactions in the network) change the parameters values.

\section{Limitations of this study and future works}

In this study, we did not explicitly address the important issue of interrelating the two described models as this issue would deserve a specific study itself. In particular, it may be interesting to investigate whether mathematical approaches (equation-free, statistical neurodynamics, aggregation of variables) can help us i) to reduce the detailed model into an aggregated model, ii) to identify the macrodynamic variables and equations and iii) to determine to what extent these variables and equations correspond to those governing the lumped-parameter model.

This work is still to be done. What we can do, at this stage, is to establish some qualitative relations between both models. First, since the two models are intended to reproduce the same neuronal system (the hippocampal CA1 sub-field), they share a number of features. Both models include main pyramidal cells and different types of interneurons, although the detailed model adds a subset of bistratified cells which is not presented in the lumped model. The connectivity patterns (pyr-pyr, pyr-in, in-pyr, in-in) are comparable. Regarding the synaptic 
transmission, both models include glutamatergic and GABAergic synapses. However, a distinction between AMPA and NMDA receptors is accounted for in the detailed model, conversely to the lumped model in which the average EPSP is characterized by a single kinetics. Second, both models have some limitations. At this stage, none of them implements plasticity-related effects, although these could be obtained by adding a time-dependence on some relevant parameters (for instance, the amplitude of PSPs at macro. level or the conductance values at micro. level). Interneurons are simplified and limited to two or three types. The input to CA1 is restricted to CA3. Finally, models do not account for metabolic variables nor for interactions with glial cells which may also be important in epilepsy.

Nevertheless, and despite these limitations, we could find some similarities in the LFPs generated by both models. In the microscopic model, we noticed that some model parameter configurations could lead to sustained rhythmic activity that could correspond to ictal-like activity. So far, we have not analyzed this model bifurcation into details. Therefore, at this stage, any comparison with the ictal-like activity generated by the macroscopic model would be pure speculation. Conversely, for epileptic spikes, we could simulate quite similar "LFP events" from both models. Therefore, we are in a situation where the same system (the hippocampus CA1 sub-field), modelled at two different scales (cellular and population), can produce similar spikes. In this context, the identification of some relationships between parameter sets at considered levels of description (cellular and population) seems achievable. These relationships would help us to better understand the meaning of aggregated parameters in macroscopic models as a function of microscopic ones. We think that this simulation framework is a first step toward the development of across-scale approaches and further toward multi-level models. 


\section{Acknowledgement}

This work was supported by "Region Bretagne" (CREATE 2009, « EPIGONE » project). Authors are grateful to Dr Marco de Curtis for providing the data recorded from the Guinea pig isolated brain preparation. 


\section{References}

Albus, K., Wahab, A. \& Heinemann, U. (2008) Standard antiepileptic drugs fail to block epileptiform activity in rat organotypic hippocampal slice cultures. $\mathrm{Br} J$ Pharmacol, 154, 709-724.

Andersen, P., Morris, R., Amaral, D., Bliss, T. \& O'Keefe, J. (2007) The Hippocampus Book, New York.

Asano, E., Muzik, O., Shah, A., Juhasz, C., Chugani, D.C., Sood, S., Janisse, J., Ergun, E.L., Ahn-Ewing, J., Shen, C., Gotman, J. \& Chugani, H.T. (2003) Quantitative interictal subdural EEG analyses in children with neocortical epilepsy. Epilepsia, 44, 425-434.

Avoli, M., Biagini, G. \& de Curtis, M. (2006) Do Interictal Spikes Sustain Seizures and Epileptogenesis? Epilepsy Curr., 6, 203-207.

Avoli, M. \& de Curtis, M. (2011) GABAergic synchronization in the limbic system and its role in the generation of epileptiform activity. Prog Neurobiol, 95, 104-132.

Bancaud, J. \& Talairach, J. (1973) [Methodology of stereo EEG exploration and surgical intervention in epilepsy]. Rev Otoneuroophtalmol, 45, 315-328.

Banks, M.I., White, J.A. \& Pearce, R.A. (2000) Interactions between distinct GABA(A) circuits in hippocampus. Neuron, 25, 449-457.

Bartolomei, F., Chauvel, P. \& Wendling, F. (2008) Epileptogenicity of brain structures in human temporal lobe epilepsy: a quantified study from intracerebral EEG. Brain, 131, 1818-1830.

Bernard, C., Esclapez, M., Hirsch, J.C. \& Ben-Ari, Y. (1998) Interneurones are not so dormant in temporal lobe epilepsy: a critical reappraisal of the dormant basket cell hypothesis. Epilepsy Res, 32, 93-103.

Bouilleret, V., Ridoux, V., Depaulis, A., Marescaux, C., Nehlig, A. \& Le Gal La Salle, G. (1999) Recurrent seizures and hippocampal sclerosis following intrahippocampal kainate injection in adult mice: electroencephalography, histopathology and synaptic reorganization similar to mesial temporal lobe epilepsy. Neuroscience, 89, 717-729.

Bragin, A., Engel, J., Jr., Wilson, C.L., Fried, I. \& Buzsaki, G. (1999a) High-frequency oscillations in human brain. Hippocampus, 9, 137-142.

Bragin, A., Engel, J., Jr., Wilson, C.L., Fried, I. \& Mathern, G.W. (1999b) Hippocampal and entorhinal cortex high-frequency oscillations $(100--500 \mathrm{~Hz})$ in human epileptic brain and in kainic acid--treated rats with chronic seizures. Epilepsia, 40, 127-137.

Cohen, I., Navarro, V., Clemenceau, S., Baulac, M. \& Miles, R. (2002) On the origin of interictal activity in human temporal lobe epilepsy in vitro. Science, 298, 1418-1421.

Coombes, S. (2010) Large-scale neural dynamics: simple and complex. Neuroimage, 52, 731739.

Cossart, R., Dinocourt, C., Hirsch, J.C., Merchan-Perez, A., De Felipe, J., Ben-Ari, Y., Esclapez, M. \& Bernard, C. (2001) Dendritic but not somatic GABAergic inhibition is decreased in experimental epilepsy. Nat Neurosci, 4, 52-62.

Deco, G., Jirsa, V.K., Robinson, P.A., Breakspear, M. \& Friston, K. (2008) The dynamic brain: from spiking neurons to neural masses and cortical fields. PLoS Comput Biol, 4, e1000092.

Demont-Guignard, S., Benquet, P., Gerber, U., Biraben, A., Martin, B. \& Wendling, F. (2011) Distinct hyperexcitability mechanisms underlie fast ripples and epileptic spikes. Ann Neurol in press.

Demont-Guignard, S., Benquet, P., Gerber, U. \& Wendling, F. (2009) Analysis of intracerebral EEG recordings of epileptic spikes: insights from a neural network model. IEEE Trans Biomed Eng, 56, 2782-2795. 
Dyhrfjeld-Johnsen, J., Santhakumar, V., Morgan, R.J., Huerta, R., Tsimring, L. \& Soltesz, I. (2007) Topological determinants of epileptogenesis in large-scale structural and functional models of the dentate gyrus derived from experimental data. J Neurophysiol, 97 , 1566-1587.

Fisher, R.S., Webber, W.R., Lesser, R.P., Arroyo, S. \& Uematsu, S. (1992) High-frequency EEG activity at the start of seizures. J Clin Neurophysiol, 9, 441-448.

Freeman, W.J. (1973) A model of the olfactory system. In M. A. B. Brazier, D. O. Walter, \& D. Schneider (Eds.), Neural modeling, pp. 41-62. Los Angeles: Univ. of California.

Freeman, W.J. (1978) Models of the dynamics of neural populations. Electroencephalogr Clin Neurophysiol Suppl, 9-18.

Gahwiler, B.H., Capogna, M., Debanne, D., McKinney, R.A. \& Thompson, S.M. (1997) Organotypic slice cultures: a technique has come of age. Trends Neurosci, 20, 471-477.

Geselowitz, D.B. (1967) On bioelectric potentials in an inhomogeneous volume conductor. Biophys $J, 7,1-11$.

Gnatkovsky, V., Librizzi, L., Trombin, F. \& de Curtis, M. (2008) Fast activity at seizure onset is mediated by inhibitory circuits in the entorhinal cortex in vitro. Ann Neurol, 64, 674-686.

Hajos, M., Hoffmann, W.E., Orban, G., Kiss, T. \& Erdi, P. (2004) Modulation of septohippocampal Theta activity by GABAA receptors: an experimental and computational approach. Neuroscience, 126, 599-610.

Hodgkin, A.L. \& Huxley, A.F. (1952) The components of membrane conductance in the giant axon of Loligo. $J$ Physiol, 116, 473-496.

Hodgkin, A.L., Huxley, A.F. \& Katz, B. (1952) Measurement of current-voltage relations in the membrane of the giant axon of Loligo. J Physiol, 116, 424-448.

Hoffman, D.A., Magee, J.C., Colbert, C.M. \& Johnston, D. (1997) K+ channel regulation of signal propagation in dendrites of hippocampal pyramidal neurons. Nature, 387, 869875.

Hufnagel, A., Dumpelmann, M., Zentner, J., Schijns, O. \& Elger, C.E. (2000) Clinical relevance of quantified intracranial interictal spike activity in presurgical evaluation of epilepsy. Epilepsia, 41, 467-478.

Huneau, C., Demont-Guignard, S., Benquet, P., Martin, B. \& Wendling, F. (2010) Timedomain features of epileptic spikes as potential bio-markers of the epileptogenesis process. Conf Proc IEEE Eng Med Biol Soc, 2010, 6007-6010.

Ibarz, J.M., Foffani, G., Cid, E., Inostroza, M. \& Menendez de la Prida, L. (2010) Emergent dynamics of fast ripples in the epileptic hippocampus. J Neurosci, 30, 16249-16261.

Jacobs, J., Levan, P., Chatillon, C.E., Olivier, A., Dubeau, F. \& Gotman, J. (2009) High frequency oscillations in intracranial EEGs mark epileptogenicity rather than lesion type. Brain, 132, 1022-1037.

Jacobs, J., Zijlmans, M., Zelmann, R., Chatillon, C.E., Hall, J., Olivier, A., Dubeau, F. \& Gotman, J. (2010) High-frequency electroencephalographic oscillations correlate with outcome of epilepsy surgery. Ann Neurol, 67, 209-220.

Klausberger, T. \& Somogyi, P. (2008) Neuronal diversity and temporal dynamics: the unity of hippocampal circuit operations. Science, 321, 53-57.

Labyt, E., Frogerais, P., Uva, L., Bellanger, J.J. \& Wendling, F. (2007) Modeling of entorhinal cortex and simulation of epileptic activity: insights into the role of inhibitionrelated parameters. IEEE Trans Inf Technol Biomed, 11, 450-461.

Lancaster, B. \& Adams, P.R. (1986) Calcium-dependent current generating the afterhyperpolarization of hippocampal neurons. J Neurophysiol, 55, 1268-1282.

Lancaster, B. \& Nicoll, R.A. (1987) Properties of two calcium-activated hyperpolarizations in rat hippocampal neurones. J Physiol, 389, 187-203. 
Lopes da Silva, F.H., Hoeks, A., Smits, H. \& Zetterberg, L.H. (1974) Model of brain rhythmic activity. The alpha-rhythm of the thalamus. Kybernetik, 15, 27-37.

Lytton, W.W. (2008) Computer modelling of epilepsy. Nat Rev Neurosci, 9, 626-637.

Marsh, E.D., Peltzer, B., Brown, M.W., 3rd, Wusthoff, C., Storm, P.B., Jr., Litt, B. \& Porter, B.E. (2010) Interictal EEG spikes identify the region of electrographic seizure onset in some, but not all, pediatric epilepsy patients. Epilepsia, 51, 592-601.

Mayer, M.L. \& Westbrook, G.L. (1983) A voltage-clamp analysis of inward (anomalous) rectification in mouse spinal sensory ganglion neurones. J Physiol, 340, 19-45.

Molaee-Ardekani, B., Benquet, P., Bartolomei, F. \& Wendling, F. (2010a) Computational modeling of high-frequency oscillations at the onset of neocortical partial seizures: From 'altered structure' to 'dysfunction'. Neuroimage, 52, 1109-1122.

Molaee-Ardekani, B., Benquet, P., Bartolomei, F. \& Wendling, F. (2010b) Computational modeling of high-frequency oscillations at the onset of neocortical partial seizures: from 'altered structure' to 'dysfunction'. Neuroimage, 52, 1109-1122.

Muhlethaler, M., de Curtis, M., Walton, K. \& Llinas, R. (1993) The isolated and perfused brain of the guinea-pig in vitro. Eur J Neurosci, 5, 915-926.

Murakami, S. \& Okada, Y. (2006) Contributions of principal neocortical neurons to magnetoencephalography and electroencephalography signals. $J$ Physiol, 575, 925-936.

Pinsky, P.F. \& Rinzel, J. (1994) Intrinsic and network rhythmogenesis in a reduced Traub model for CA3 neurons. J Comput Neurosci, 1, 39-60.

Rodin, E., Constantino, T., Rampp, S. \& Wong, P.K. (2009) Spikes and epilepsy. Clin EEG Neurosci, 40, 288-299.

Soltesz, I. \& Staley, K.J. (2008) Computational Neuroscience in Epilepsy. Elsevier.

Staley, K.J., White, A. \& Dudek, F.E. (2011) Interictal spikes: harbingers or causes of epilepsy? Neurosci Lett, 497, 247-250.

Stefan, H., Lopes da Silva, F.H., Loscher, W., Schmidt, D., Perucca, E., Brodie, M.J., Boon, P.A., Theodore, W.H. \& Moshe, S.L. (2006) Epileptogenesis and rational therapeutic strategies. Acta Neurol Scand, 113, 139-155.

Suffczynski, P., Kalitzin, S., Pfurtscheller, G. \& Lopes da Silva, F.H. (2001) Computational model of thalamo-cortical networks: dynamical control of alpha rhythms in relation to focal attention. Int $J$ Psychophysiol, 43, 25-40.

Suffczynski, P., Wendling, F., Bellanger, J.-J. \& Da Silva, F.H.L. (2006) Some insights into computational models of (Patho)physiological brain activity. Proceedings of the IEEE, 94, 784- 804.

Suzuki, F., Junier, M.P., Guilhem, D., Sorensen, J.C. \& Onteniente, B. (1995) Morphogenetic effect of kainate on adult hippocampal neurons associated with a prolonged expression of brain-derived neurotrophic factor. Neuroscience, 64, 665-674.

Talairach, J. \& Bancaud, J. (1966) Lesion, "irritative" zone and epileptogenic focus. Confin Neurol, 27, 91-94.

Taylor, A.L., Goaillard, J.M. \& Marder, E. (2009) How multiple conductances determine electrophysiological properties in a multicompartment model. J Neurosci, 29, 55735586.

Thompson, S., Cai, X., Dinocourt, C. \& Nestor, M. (2005) The Use of Brain Slice Cultures for the Study of Epilepsy. in Models of Seizures and Epilepsy, Eds : A. Pitkänen, P.A. Schwartzkroin, S.L. Moshé, Elsevier Academic Press, Burlington, San Diego, London.

Traub, R.D. (1979) Neocortical pyramidal cells: a model with dendritic calcium conductance reproduces repetitive firing and epileptic behavior. Brain Res, 173, 243-257.

Ullah, G. \& Schiff, S. (2009) Models of epilepsy. Scholarpedia (http://www.scholarpedia.org/article/Models_of epilepsy), 4(7), 1409. 
van Rotterdam, A., Lopes da Silva, F.H., van den Ende, J., Viergever, M.A. \& Hermans, A.J. (1982) A model of the spatial-temporal characteristics of the alpha rhythm. Bull Math Biol, 44, 283-305.

Wendling, F. (2008) Computational models of epileptic activity: a bridge between observation and pathophysiological interpretation. Expert Rev Neurother, 8, 889-896.

Wendling, F., Bartolomei, F., Bellanger, J.J., Bourien, J. \& Chauvel, P. (2003) Epileptic fast intracerebral EEG activity: evidence for spatial decorrelation at seizure onset. Brain, 126, 1449-1459.

Wendling, F., Bartolomei, F., Bellanger, J.J. \& Chauvel, P. (2002) Epileptic fast activity can be explained by a model of impaired GABAergic dendritic inhibition. Eur J Neurosci, 15, 1499-1508.

Wendling, F. \& Chauvel, P. (2008a) Transition to ictal activity in temporal lobe epilepsy: insights from macroscopic models. In: Soltesz, I., Staley, K. (Eds), "Computational Neuroscience in Epilepsy", Academic Press, pp. 356-386.

Wendling, F. \& Chauvel, P. (2008b) Transition to ictal activity in Temporal Lobe Epilepsy: insights from macroscopic models. Computational Neuroscience in Epilepsy, Edited by Drs. Ivan Soltesz and Kevin Staley, Elsevier.

Wendling, F., Hernandez, A., Bellanger, J.J., Chauvel, P. \& Bartolomei, F. (2005) Interictal to ictal transition in human temporal lobe epilepsy: insights from a computational model of intracerebral EEG. J Clin Neurophysiol, 22, 343-356.

White, A., Williams, P.A., Hellier, J.L., Clark, S., Edward Dudek, F. \& Staley, K.J. (2010) EEG spike activity precedes epilepsy after kainate-induced status epilepticus. Epilepsia, 51, 371-383.

White, J.A., Banks, M.I., Pearce, R.A. \& Kopell, N.J. (2000) Networks of interneurons with fast and slow gamma-aminobutyric acid type A (GABAA) kinetics provide substrate for mixed gamma-theta rhythm. Proc Natl Acad Sci U S A, 97, 8128-8133.

Wilson, H.R. \& Cowan, J.D. (1972) Excitatory and inhibitory interactions in localized populations of model neurons. Biophys $J, 12,1-24$.

Worrell, G. \& Gotman, J. (2011) High-frequency oscillations and other electrophysiological biomarkers of epilepsy: clinical studies. Biomark Med, 5, 557-566.

Zijlmans, M., Jiruska, P., Zelmann, R., Leijten, F., Jefferys, J. \& Gotman, J. (2011) High frequency oscillations as a new biomarker in epilepsy. Ann Neurol, in press. 


\section{Legends}

Figure 1: Epileptic spikes and Fast Ripples (FRs). (A) Human (hippocampus), in vivo (mouse, hippocampus) and in vitro (rat organotypic hippocampal slice) interictal spikes recorded with extracellular electrodes. Simulated local field potentials (LFPs) generated from microscopic (cellular level) and macroscopic (neuronal population level) computational models of the hippocampus CA1 subfield. (B) Human (hippocampus), in vivo (mouse, hippocampus) and in vitro (rat organotypic hippocampal slice) FRs recorded with extracellular electrodes. Simulated local field potentials (LFPs) generated by the macroscopic model. Color-coded maps are time-frequency (TF) representations (spectrograms) of corresponding signals. Hot (resp. cold) colors correspond to the presence (resp. absence) of signal energy at a given time ( $\mathrm{X}$ axis) and frequency (Y axis) point in the TF plane.

Figure 2: Electrophysiological signals recorded during the interictal to ictal transition. (A) Depth-EEG signal recorded from hippocampus in a patient with temporal lobe epilepsy (TLE). (B) Local field potential (LFP) recorded in a freely-moving chronic epileptic mouse with micro-electrodes implanted in hippocampus, 4 weeks after the Kainic acid (KA) injection. (C) LFP recorded with intra-hippocampal electrode in an isolated brain preparation (guinea pig) after transient arterial perfusion of bicuculline. (D) LFP simulated with the macroscopic model when inhibition-related parameter is gradually decreased as a function of time. Total durations of signals shown in A, B and C is 120 seconds. Duration of signal D is 60 seconds. (E) Time-frequency diagrams focusing on the fast onset activity (tonic phase of the seizure) for upper signals.

Figure 3: Computational models intended to reproduce the hippocampal activity (CA1 subfield). (A) Lumped-parameter model of CA1. The neuronal population was assumed to 
consist of three sub-populations of neurons corresponding to main (pyramidal) cells and to two types of local inhibitory interneurons (OLM and basket cells) projecting to either the peri-dendritic or the peri-somatic region of pyramidal cells. Input to interneurons is excitatory (Glutamatergic). Feedback to pyramidal cells is either excitatory (Glutamatergic) or inhibitory (GABAergic). Slow (GABAA,slow) and fast (GABAA,fast) kinetics are associated to inhibitory post-synaptic potentials (PSP) depending on the location of GABA receptors, either in the peri-dendritic or in the peri-somatic region of pyramidal cells. (B) Detailed model in which each cell is explicitly represented (CA1 pyramidal cell: two-compartment model. OLM, bistratified basket cells: one-compartment model). The CA1 pyramidal cell was created based on a precise selection of distinct types of dendritic and somatic ionic channel, according to the literature. Physiological properties of ionic channels such as kinetic of activation and inactivation, voltage dependence and reversal potential were respected. Single neuron models are synaptically (AMPA-, NMDA- and GABA-ergic synapses) interconnected into network (about 800 neurons and 200 interneurons). To mimic the excitatory input from CA3 to CA1, the network model was stimulated with an afferent volley of APs coming from an "external array of cells".

Figure 4: Macroscopic (lumped-parameter) model. Simulation of the transition from interictal to ictal activity. For increased excitation (amplitude of average EPSPs) and gradual decrease of inhibition (amplitude of average IPSPs), dramatic changes are observed in simulated LFPs. (A) Background activity. (B) Sporadic spikes. (C) Fast onset activity. (D) Rhythmic ictal activity.

Figure 5: Microscopic (detailed) model. The number and spatial distribution of involved pyramidal cells, their degree of excitability and the network synchronization are critical 
factors that strongly modify the morphology of epileptic events induced in the LFP. (A) Field EPSP simulated for "normal" conditions. (B) FR simulated for altered excitability conditions (small cluster of hyperexcitable pyramidal cells, slightly increased excitation and slightly decreased inhibition). (C) Epileptic spike simulated for another alteration of excitability conditions (large cluster of hyperexcitable pyramidal cells, markedly increased excitation and decreased inhibition).

Figure 6: Macroscopic model of hippocampal activity. The fast onset acitivity (FOA) observed at the onset of seizures is explained by the sustained firing of somatic-projecting interneurons (fast $\mathrm{GABA}_{\mathrm{A}}$ recepetor-mediated IPSPs). During this FOA, the average firing rate in the pyramidal cell sub-population is dramatically reduced. Experimental validation of these findings were reported in (Gnatkovsky et al., 2008) although authors focused on the entorhinal cortex.

Figure 7: Experimental validation of the detailed model predictions. (A) In vitro recordings were performed to test the model prediction about the effect of network excitability (respective role of glutamatergic and GABAeric neurotransmission) on the generation of distinct interictal (spikes or fast ripples - FRs -) and ictal-like activity. (B) Under control condition (normal excitability), no FRs nor epileptic spikes could be generated. (C) As predicted by the model, evoked FRs could be generated with a moderate increase of excitability while (D) Epileptic spikes could be obtained when excitability was significantly increased. (E) Further increase of excitability induced seizure-like activity. CA1 responses were induced by electrical stimulation of the CA3-CA1 pathway. Increase of excitability was obtained by the lowering magnesium level (unblocked NMDA receptors) and adding low concentration of $\mathrm{GABA}_{\mathrm{A}}$ receptor antagonist $(2-4 \mu \mathrm{M}$ bicuculline). Evoked epileptic spikes 
could be generated by adding AMPA $(4-6 \mu \mathrm{M})$ to the previous condition. Transient seizurelike activity followed by spontaneous epileptic spikes could be obtained by using either 4AP $(100 \mu \mathrm{M})$ or high concentration of bicuculline $(40 \mu \mathrm{M})$. EC: extracellular. IC: intracellular. 
Figure 1

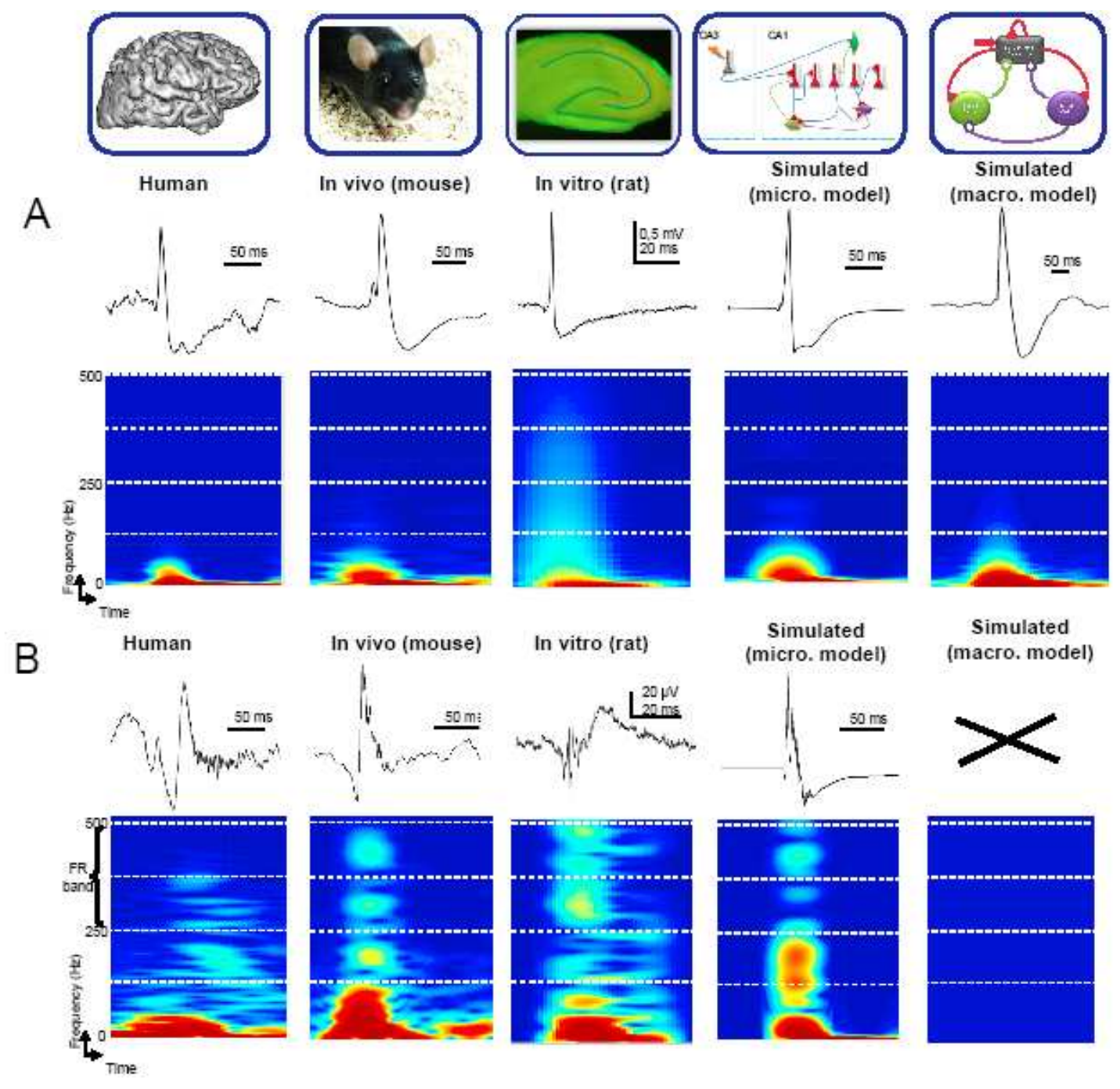


Figure 2

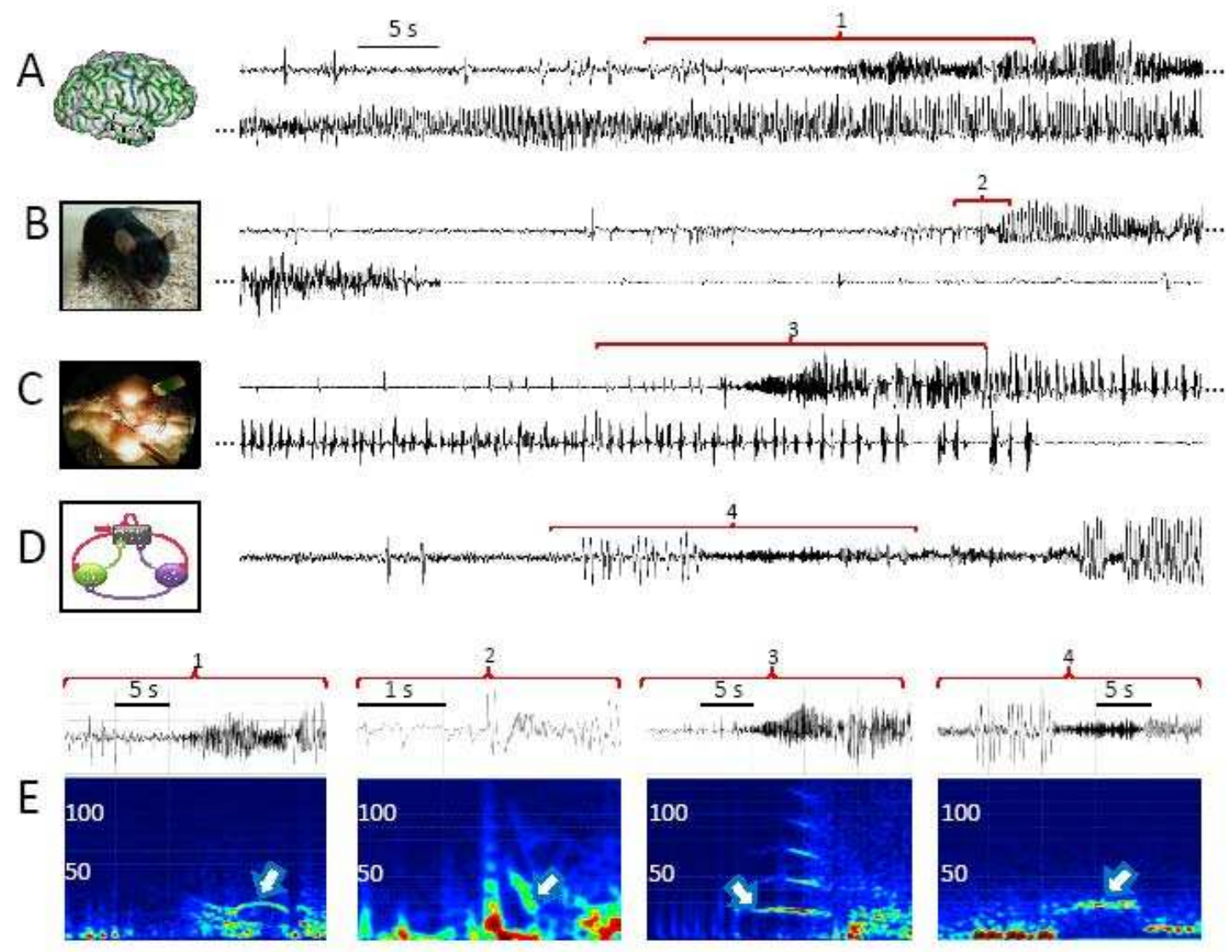


Figure 3

A. Lumped-parameter model
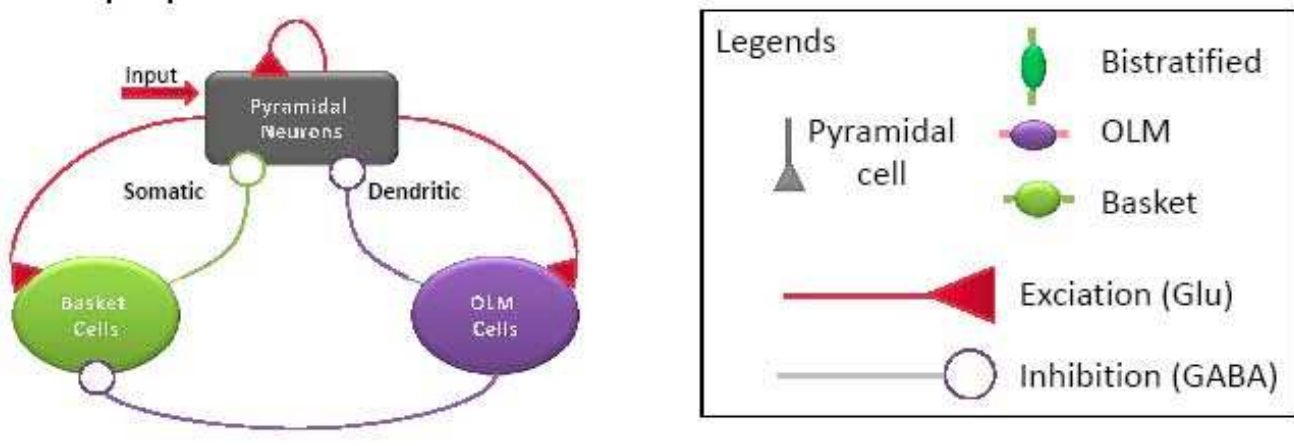

B. Detailed model
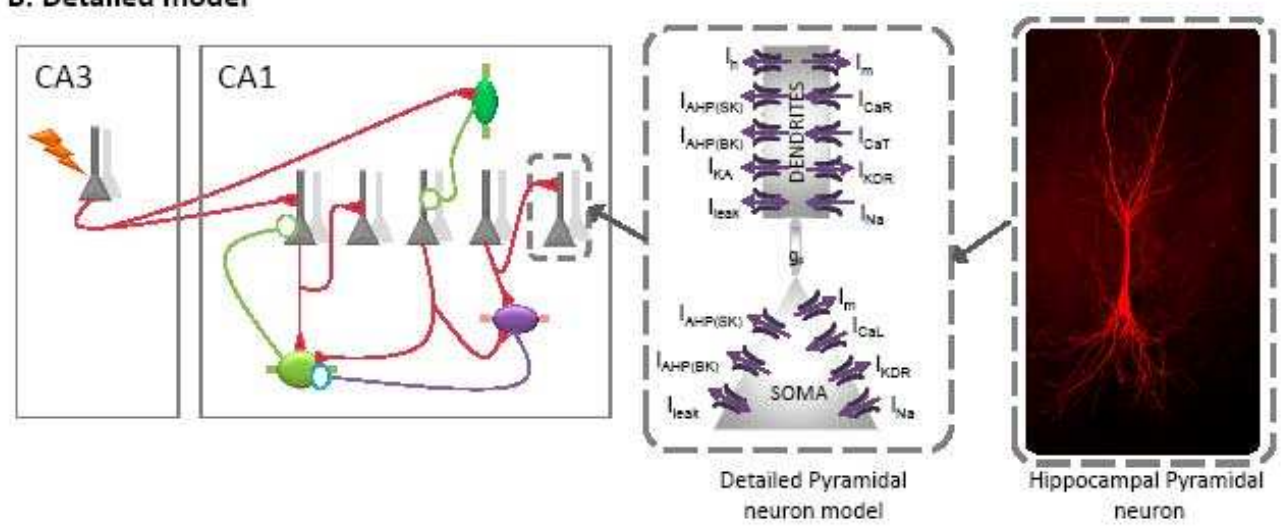
Figure 4

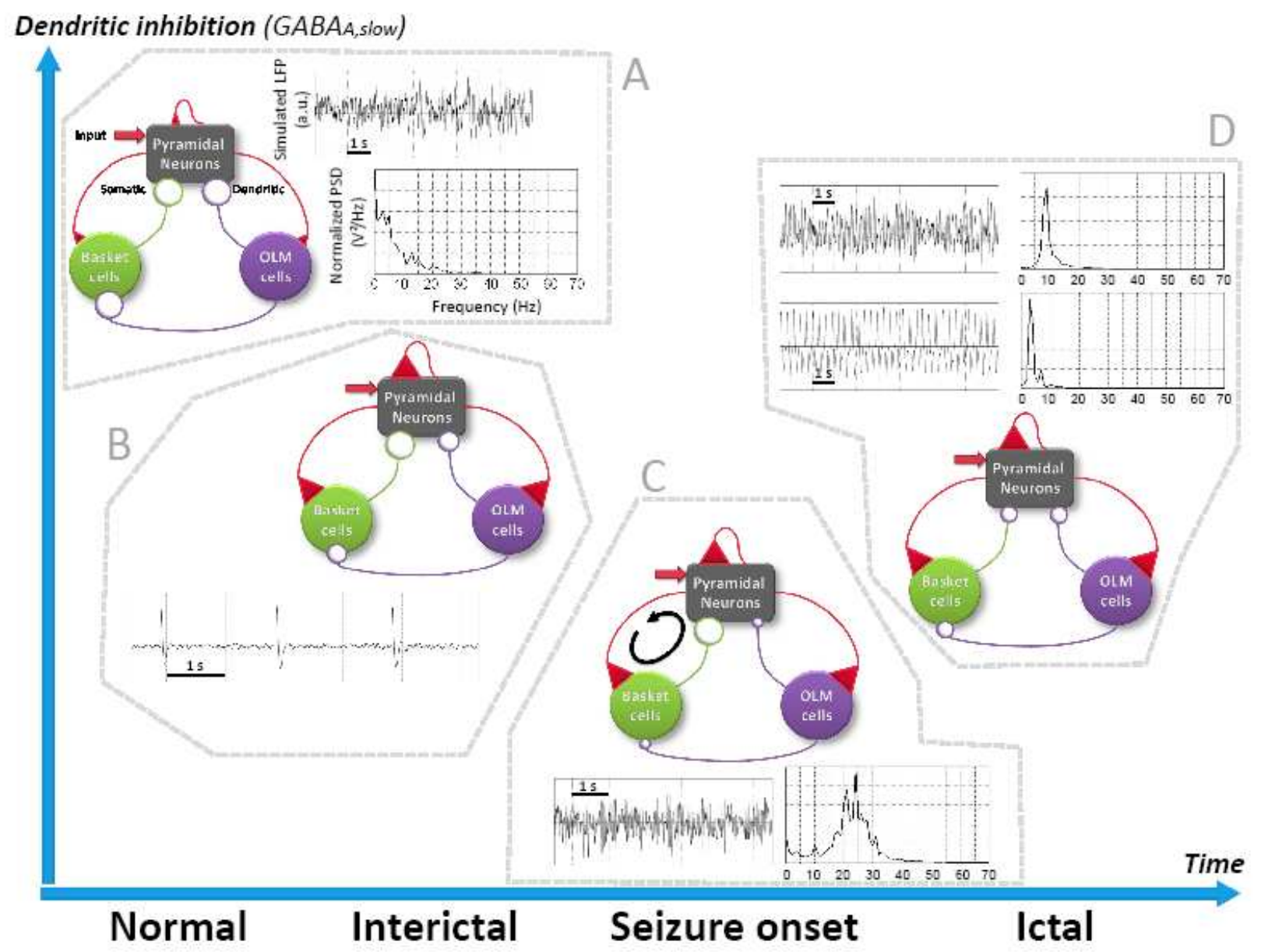


Figure 5

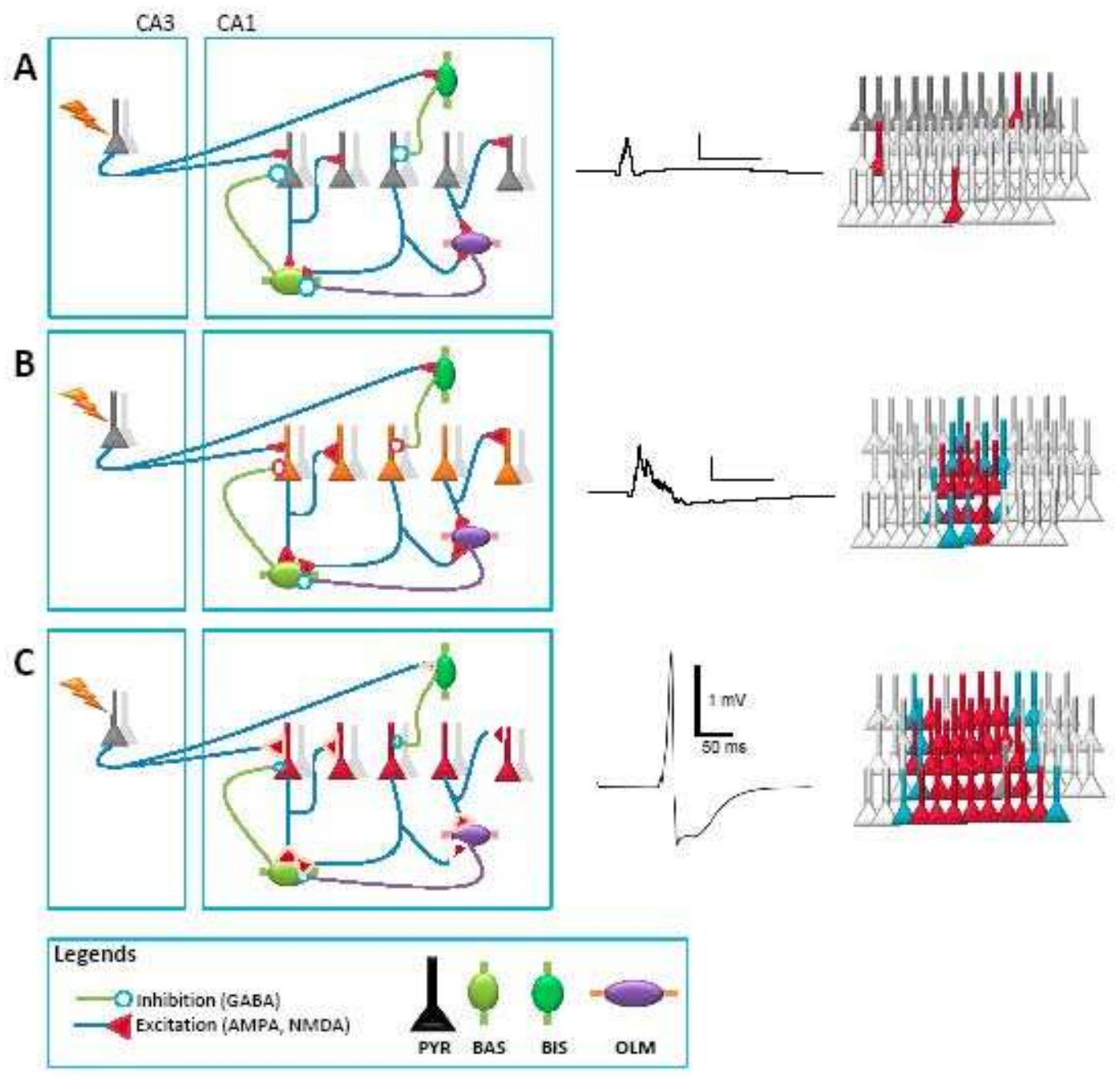


Figure 6

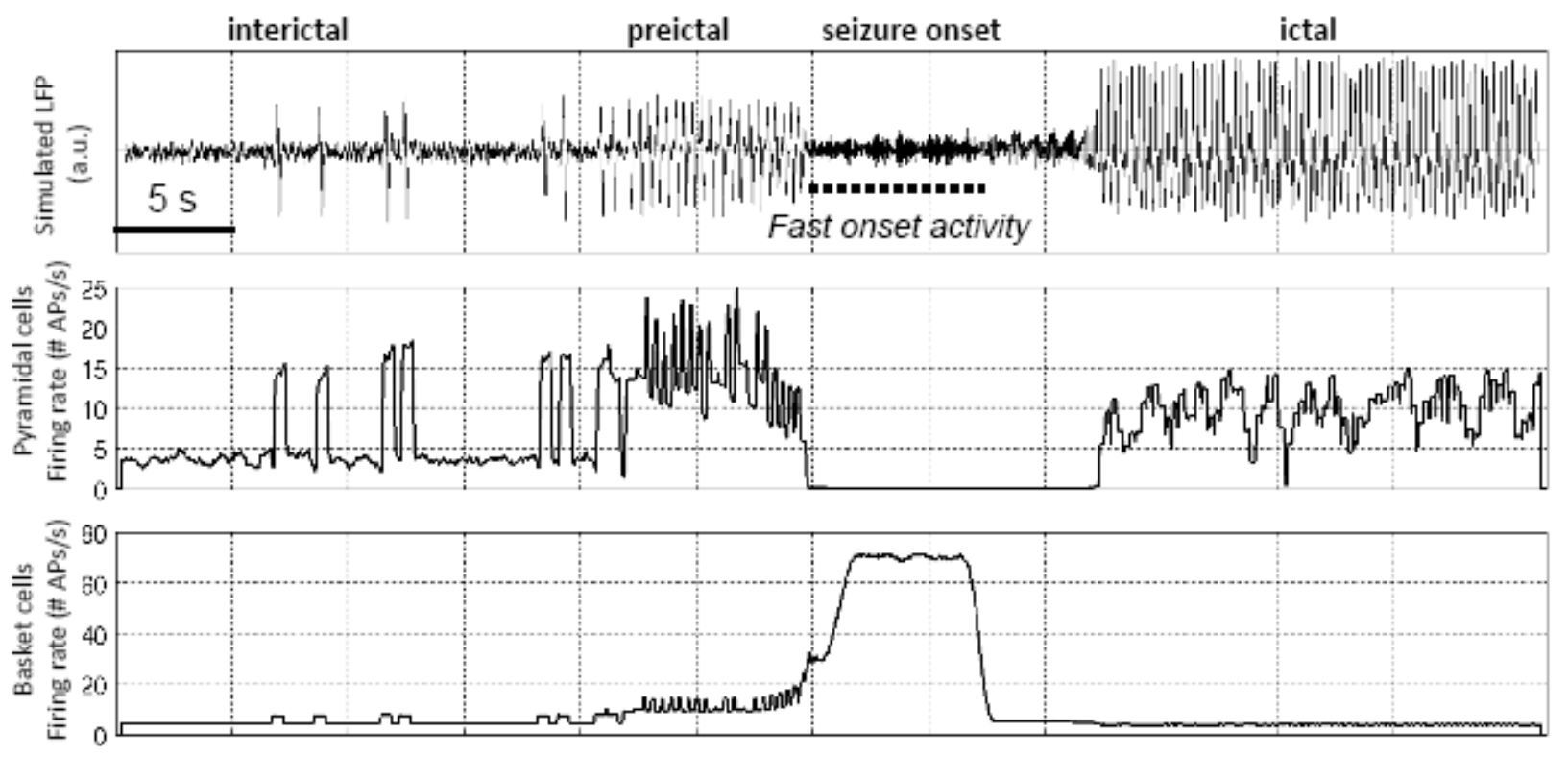


Figure 7

Decrease of

GABAergic inhibition

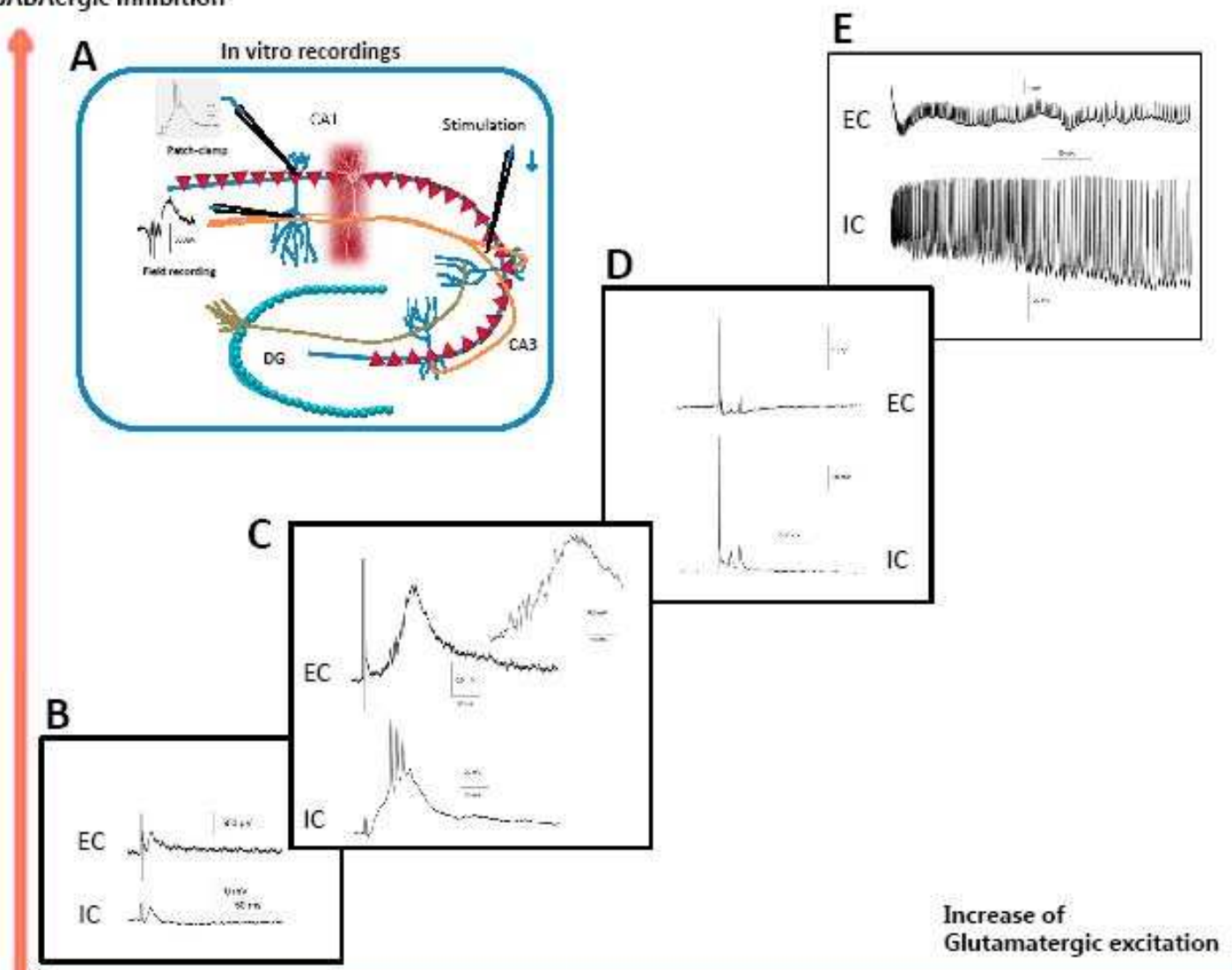

